\title{
REDES DE COOPERAÇÃO: UMA PROPOSTA DE ESCALA QUANTITATIVA
}

Marjulie Alberton ${ }^{1}$

Júlio César Da Silva ${ }^{1}$

Carlos Eduardo Carvalho ${ }^{2}$

${ }^{1}$ Fundação Universidade Regional de Blumenau (FURB)

${ }^{2}$ Universidade do Oeste de Santa Catarina (UNOESC) 


\section{REDES DE COOPERAÇÃO UMA PROPOSTA DE ESCALA QUANTITATIVA}

Resumo: O interesse na pesquisa sobre redes de cooperação interorganizacionais incita a necessidade de mensurar fatores relacionados ao tema. Após uma revisão de literatura, não se encontrou escalas quantitativas que mensurassem itens especificamente relacionados. Entrevistas semiestruturadas ou questionários de escalas, aplicado em pesquisas anteriores, não atende as necessidades de pesquisadores que querem realizar um estudo quantitativo sobre o tema e observar fatores relacionados. Assim, surgiu a necessidade de desenvolver tal escala, baseada em itens encontrados na literatura: cooperação, confiança, transferência de conhecimentos, relações sociais. Esta pesquisa possui caráter descritivo, tipo survey e corte transversal. Após validação, aplicou-se a escala em 125 empresas manufatureiras de pequeno e médio porte no Brasil. Para análise, utilizou-se o software SmartPLS, no qual realizou-se o teste de confiabilidade do constructo, teste-t e $p$-value das assertivas. O resultado aponta para item confiança como o mais forte, enquanto transferência de conhecimento é o mais fraco dentre itens estudados.

Palavras-chave: Cooperação. Confiança. Transferência de Conhecimento. Relações Sociais.

\section{$1 \quad$ Introdução}

O desempenho organizacional é o que faz com que as organizações se destaquem dentre tantas, assim, a busca pelo desempenho de excelência pode ser um desafio às organizações, mas também um vislumbre de oportunidades. De acordo com Hoskisson, Hitt, Wan e Yiu (1999), a busca pelo desempenho organizacional é atividade essencial da administração, e a área da estratégia tem se preocupado historicamente em explicar diferenças de desempenho entre as empresas. A abordagem da visão baseada em recursos tem proposto que as diferenças de desempenho surgem da heterogeneidade entre as firmas. Assim, considerando que cada organização possui forças e fraquezas, é relevante identificá-las de modo a distingui-las (WERNERFELT, 1984). Fatores individuais, organizacionais e ambientais podem influenciar na melhora ou piora da capacidade de inovação de uma organização (DAMANPOUR; SZABAT; EVAN, 1989; DAMANPOUR, 1991).

Dentre várias estratégias possíveis, a participação de empresas em redes de cooperação interorganizacionais, configuram uma forma inovativa de conquistar competitividade. A associação entre organizações para a atuação conjunta, é uma possibilidade real ao desenvolvimento organizacional (OLAVE; NETO, 2001). Essa associação, muitas vezes ocorre por afinidade e de modo informal entre as organizações, o que as deixa responsáveis pelo seu próprio desenvolvimento (OLAVE; NETO, 2001; PROVAN; FISH; SYDOW, 2007). A literatura aborda diversos benefícios pertinentes às organizações que constituem uma rede de cooperação, como por exemplo, a resolução conjunta de problemas (MOLINA-MORALES et al., 2015), o compartilhamento de informações e conhecimentos (BALESTRIN; VARGAS, 2004), o aumento da confiança e do comportamento cooperativo (ANDRIGHI; HOFFMANN; ANDRADE, 2011), a definição de estratégias organizacionais (THORGREN; WINCENT; ÖRTQVIST, 2009) e dentre outros tantos benefícios, a consequentemente melhoria no desempenho organizacional (MOLINA-MORALES et al., 2015). Por outro lado, verifica-se 
estudos que levantam o não benefício da participação em redes, como a influência negativa dos laços de cooperação no desempenho inovador (STROBEL; KRATZER, 2017), e os riscos e custos elevados que atrapalham a cooperação entre as organizações (CONSENTINO; CARVALHO; GOULART, 2017).

Embora diversos estudos abordam o tema redes de cooperação, nos mais variados contextos e relações (DIAS; FRANCO, 2018, YOU; SHU; LUO, 2017; ROLT; DIAS; PEÑA, 2017; VERSCHOORE; BALESTRIN; TEIXEIRA, 2017; ANDRADE; FERREIRA; VERONEZE; CAVAZZA; SANTOS, 2017, CONSENTINO; PAIVA DE CARVALHO; GOULART, 2017), não se encontrou estudos que apresentem uma escala quantitativa sobre redes de cooperação interorganizacionais. Diante deste contexto, este trabalho visa o desenvolvimento de uma escala que mensure quantitativamente, itens relacionados a redes de cooperação interorganizacionais. Ainda, busca-se testar a validade e confiabilidade da escala de mensuração proposta. O desenvolvimento da escala baseou-se, principalmente, em Hoffmann et al. (2017), que considera itens relacionados às redes de cooperação interorganizacionais como cooperação, confiança, transferência de conhecimento e relações sociais. Pretende-se assim, contribuir com a validação de uma proposta quantitativa, que considere os itens relacionados às redes de cooperação interorganizacionais. A escala poderá ser utilizada em estudos que necessitem de medida subjetiva, ou seja, que consideram a percepção do respondente, podendo ser aplicada em empresas, instituições e universidades.

A estrutura do trabalho apresenta inicialmente a fundamentação teórica relativo às redes de cooperação. Segue-se os procedimentos metodológicos do desenvolvimento da escala, bem como, testes de validade e confiabilidade. Apresentam-se os resultados e finalmente, breve consideração destes.

\section{$2 \quad$ Referencial Teórico}

As redes são formadas por um grupo de organizações que estão interligadas por alguma forma de interação e algum grau de convergência (FRANCO; BELO, 2013). Em âmbito mundial, o movimento de mudanças nas relações industriais se intensifica a partir dos anos 80 à medida que se acumulam e se consolidam as transformações técnicas, organizacionais e econômicas. Essas mudanças causam alterações na maneira de produção, de administração e de distribuição. Como consequência, essas alterações criam novas relações entre empresas, entre empresas e trabalhadores e entre empresas e instituições (OLAVE; NETO, 2001).

Surge então, como possibilidade para o desenvolvimento organizacional, as diversas formas de relações entre empresas, como joint ventures, alianças estratégicas, relações de terceirização, distritos industriais, consórcios, redes de cooperação (GRANDORI; SODA, 1995; OLAVE; NETO, 2001), aglomerações (clusters), cadeias de suprimentos, arranjos produtivos locais, dentre outras (ANDRIGHI; HOFFMANN, 2010). Para Olave e Neto (2001) e Balestrin e Vargas (2004), as redes de empresas configuram uma forma inovativa de conquistar competitividade e sobrevivência.

Em função da variedade de formas que uma rede de empresas pode adotar, não existe uma regra única em relação às vantagens que sua constituição pode proporcionar (OLAVE; NETO, 2001). Para Powell (1990), as empresas escolhem a localização em determinada área, de acordo com a existência de densa sobreposição de aglomerados de organizações, abastecida de mão-de-obra e infraestrutura, objetivando assim, as vantagens da aglomeração. Outros 
fatores considerados pelas organizações são as questões relacionadas ao fator humano, o ambiente e as características da empresa. Quanto mais diversificada a formação da rede, mais ligações e elos são desenvolvidos, sendo mais complexas as suas relações. A rede é fortalecida por meio de estruturas heterogêneas, com diferentes tipos de atores, tornando-a cada vez mais autossuficiente (CUNHA; PASSADOR; PASSADOR, 2012).

As redes, de acordo com Powell, Koput e Smith-Doerr (1996), Ahuja (2000) e Balestrin e Vargas (2004), são um meio para a troca de recursos difíceis de medir, como o know-how, a tecnologia, a capacidade tecnológica, o estilo de produção, o conhecimento, as habilidades, o espírito de inovação ou experimentação. Já Thorgren, Wincent e Örtqvist (2009) citam que a confiança, a diversidade, o relacionamento e a transferência de conhecimento são alguns dos recursos de redes que funcionam bem.

As organizações tendem a se beneficiar com o compartilhamento de conhecimento, pois este processo pode permitir a resolução conjunta de problemas e práticas comuns de inovação (MOLINA-MORALES et al., 2015). O estudo de Balestrin e Vargas (2004), mostra que o maior ganho das organizações, por meio da formação da rede consiste no compartilhamento de informações e de conhecimentos, e que as informações compartilhadas que mais trouxeram benefícios à rede tratam de processos de produção, fornecedores, insumos, tecnologias e mercado. A transferência de conhecimento, de acordo com Hoffmann, Bandeira-de-Mello e Molina-Morales (2011) e Hoffmann et al. (2017), pode ocorrer de três formas: pela mobilidade da força de trabalho, por meio de contato social e por contato direto entre as empresas.

No entanto, alguns estudos como o de Molina-Morales e Martinez-Fernandez (2009) destacam que o aumento da intensidade nos relacionamentos não necessariamente reflete o mesmo nível de intensidade no desempenho das organizações, em termos de inovação, e Hofmann, Bandeira-de-Mello e Molina-Morales (2011) verificam que o desempenho de inovação não está diretamente relacionado com a transferência de conhecimento. Ainda, outro estudo revela que as relações de cooperação nem sempre são necessárias para a transferência de conhecimento entre organizações (HOFFMANN; LOPES; MEDEIROS, 2014).

As relações de interação social formam canais de informação que reduzem a quantidade de tempo e investimento necessário para agrupar informações. Por meio das interações com os outros, as organizações obtém acesso ao conhecimento externo, que podem articular com o conhecimento existente. Essas interações criam um contexto dentro do qual novos conhecimentos podem ser aplicados e explorados. Assim, interações sociais exercem uma influência sobre os recursos das empresas e, podem se constituir um fator explicativo de sua inovação (MOLINA-MORALES; MARTINEZ-FERNANDEZ, 2009). Por outro lado, as interações sociais envolvem custos associados à manutenção de relacionamentos existentes, pois exige tempo, dedicação, além de reuniões e troca de informações (MOLINA-MORALES; MARTINEZ-FERNANDEZ, 2007; 2009), e não devem ser associadas à um aumento paralelo em termos de resultados (MOLINA-MORALES; MARTINEZ-FERNANDEZ, 2009).

A confiança e a cooperação tendem a representar um papel central no sucesso alcançado pelas redes (BALESTRIN; VARGAS, 2004; HOFFMANN et al., 2017). Ressalta-se que o termo redes está constantemente relacionado aos comportamentos de cooperação, comunicação e confiança, como aponta Andrighi, Hoffmann e Andrade (2011). Quando dois parceiros confiam um no outro, eles tendem a compartilhar recursos sem se preocupar tanto com os comportamentos oportunistas. Algum nível de confiança interorganizacional é relevante para a 
inovação em contextos baseados em rede, pois aumenta a proximidade, a troca de informações e conhecimento tácito e consequentemente, aumenta oportunidades de acesso a novas e valiosas combinações de recursos (FELZENSZTEIN; GIMMON; CARTER, 2010; MOLINAMORALES; MARTINEZ-FERNANDEZ; TORLÒ, 2011).

Molina-Morales, Martinez-Fernandez e Torlò (2011), afirmam que a confiança é boa, porém condicional. Os autores exploram além dos impactos positivos da confiança no desempenho de inovação das organizações, e apresentam também os impactos negativos que a confiança pode gerar. O estudo de Molina-Morales, Martinez-Fernandez e Torlò (2011), mostra que aumentos adicionais de confiança trazem benefícios decrescentes e podem até diminuir os retornos de inovação para a organização.

Isso porque relacionamentos de confiança de longo prazo provavelmente chegam num ponto em que as organizações envolvidas já não trocam mais novas informações e conhecimentos, assim, o custo de manter seus relacionamentos cooperativos e de confiança pode deixar as organizações sem espaço, dinheiro, tempo ou esforço, para cultivar novos relacionamentos promissores (MOLINA-MORALES; MARTINEZ-FERNANDEZ, 2009; MOLINA-MORALES et al., 2015). Dessa forma, organizações que investem demais na confiança ou investem em relações de confiança que têm pouco valor para a empresa, podem deslocar recursos preciosos bem como assumir riscos desnecessários, que pode ter efeitos negativos sobre o desempenho de inovação. (MOLINA-MORALES; MARTINEZFERNANDEZ; TORLÒ, 2011).

A lógica baseada em recursos sugere que a vantagem competitiva de alianças é baseada na integração efetiva dos recursos valiosos entre as organizações que cooperam. Além do interesse das organizações em acessar e adquirir recursos, estas estão interessadas também em manter e melhorar os recursos que já possuem (ZONTA et al., 2015), bem como melhorar seu desempenho (MOLINA-MORALES et al., 2015). Conforme Gulati (1998), a necessidade de complementar recursos é um fator-chave para cooperação interorganizacional.

As redes de cooperação são um modo de associação por afinidade de natureza informal e que deixa cada uma das organizações responsável pelo seu próprio desenvolvimento (OLAVE; NETO, 2001; PROVAN; FISH; SYDOW, 2007). Os ganhos que as redes de cooperação podem trazer às organizações, são bastante difundidos na literatura (JÚNIOR; COSTA; HOFFMANN, 2016). As redes de cooperação estão voltadas para uma maior cooperação entre as empresas, no qual aproveitam recursos de uso comum como: tecnologia de processo, suprimentos, habilidades pessoais e organizacionais (OLAVE; NETO, 2001).

Essa cooperação, de acordo com Thorgren, Wincent e Örtqvist (2009), pode levar as organizações a obter recursos, criar combinações necessárias para introduzir novos produtos, expandir-se para novos mercados, fazer mudanças organizacionais, entre outras finalidades estratégicas. Para Zonta et al. (2015), a decisão de cooperar é uma das diversas alternativas estratégicas da organização, sendo a alternativa mais acertada para todas as ocasiões.

Assim, muitas relações de cooperação entre empresas são estabelecidas com o objetivo de minimizar dificuldades como custos de transação, isto é, os custos que vão além dos custos de produção. A cooperação ocasiona a possibilidade de dispor de tecnologias e reduzir os custos de transação relativos ao processo de inovação, aumentado a eficiência econômica e, por consequência, a competitividade (OLAVE; NETO, 2001). A cooperação entre organizações com o propósito de atingir soluções coletivas tem recebido crescente atenção nos estudos e 
práticas organizacionais, tanto no Brasil como no exterior (MOLINA-MORALES; MARTÍNEZ-FERNÁNDEZ，2007; HOFMANN; BANDEIRA-DE-MELLO; MOLINAMORALES, 2011; MOLINA-MORALES et al., 2015; ZONTA et al., 2015). Esse interesse desencadeou a busca de novas explicações e estudos para o gerenciamento de redes, sua utilidade e sua relação com o desempenho organizacional (MOLINA-MORALES; MARTÍNEZ-FERNÁNDEZ, 2007).

\section{Procedimentos Metodológicos}

Por meio da pesquisa na literaura em estudos anteriores, e a partir de Hoffmann, Bandera-de-Melo e Molina-Morales (2011), Hoffmann et al. (2017) e Zonta et al. (2015), identificou-que quatro itens relacionados às redes de cooperação, sendo eles: cooperação, confiança, transferência de conhecimento e relações sociais. Dessa forma, adaptou-se esses quatro indicadores pertinentes do estudo de Hoffmann et al. (2017), e foi desenvolvida uma escala do tipo Likert, com 7 pontos. Para verificar a precisão e a coerência da escala proposta, foram realizados testes de validade e confiabilidade.

A validação do conteúdo envolve uma avaliação sistemática por parte de especialistas para avaliar a adequação das questões ao tema (HAIR JR et al., 2005). Os indicadores iniciais sobre redes de cooperação, foram então encaminhados para oito especialistas da área do estudo, todos doutores e pesquisadores, convidando-os a revisar o pré-questionário para validar a escala quanto ao entendimento e a coerência das questões com o tema a ser pesquisado. As sugestões dos avaliadores e ajustes pertinentes estão dispostas no Quadro 1.

Quadro 1 - Sugestões e ajustes para a escala de Redes de Cooperação

\begin{tabular}{|c|l|l|}
\hline $\begin{array}{c}\text { Especia- } \\
\text { lista }\end{array}$ & \multicolumn{1}{|c|}{ Sugestão do especialista } & \multicolumn{1}{c|}{ Ajuste } \\
\hline \multirow{2}{*}{1} & $\begin{array}{l}\text { A) Sugere acrescentar "formal" no texto } \\
\text { introdutório sobre redes de cooperação. } \\
\text { B) Sugere a utilização de escala de 5 } \\
\text { pontos, alegando ser a melhor escala } \\
\text { conforme vários estudos metodológicos. }\end{array}$ & $\begin{array}{l}\text { A) Embora o foco da pesquisa está na cooperação } \\
\text { informal, não há problema em a empresa } \\
\text { participar de uma cooperação formal. Assim, a } \\
\text { sugestão foi aceita e o texto modificado. } \\
\text { B) Como o questionário utiliza outros construtos } \\
\text { já validados e com escala do tipo Likert de 7 } \\
\text { pontos, fica mais assertivo manter 7 pontos. } \\
\text { Assim, essa sugestão não foi acatada. }\end{array}$ \\
\hline 2 & $\begin{array}{l}\text { Sugere modificar a redação de uma } \\
\text { assertiva, deixando-a mais clara e objetiva. }\end{array}$ & $\begin{array}{l}\text { Assim, a sugestão foi aceita e a assertiva } \\
\text { modificada. }\end{array}$ \\
\cline { 2 - 4 } 3 & $\begin{array}{l}\text { Sugere modificar a redação das assertivas, } \\
\text { para avaliar o tipo de relação (unilateral ou } \\
\text { bilateral). }\end{array}$ & $\begin{array}{l}\text { Na presente pesquisa não se avalia o tipo de } \\
\text { relação, se é unilateral ou bilateral. Assim, essa } \\
\text { sugestão não foi acatada. }\end{array}$ \\
\hline 4 & $\begin{array}{l}\text { Sugere alterar a nomeação da escala de } \\
\text { "Muito Forte" e "Muito Fraco" para } \\
\text { "Forte" e "Fraco", alegando que a palavra } \\
\text { "muito" pode levar o respondente a não } \\
\text { assinalar os extremos, dando menor } \\
\text { variação nas respostas. }\end{array}$ & $\begin{array}{l}\text { Observação válida. Sugestão aceita e nomeação } \\
\text { de escala modificada. }\end{array}$ \\
\hline $5,6,7$ e 8 8 & \begin{tabular}{l} 
Aprovado sem sugestões. \\
\hline
\end{tabular} & \\
\hline
\end{tabular}

Fonte: Elaborado pelos autores. 
Após as sugestões e considerações dos especialistas, conforme pode-se observar no Quadro acima, foram feitas as correções cabíveis e assim, os indicadores referentes ao construto Redes de Cooperação foram aprovados pelos especialistas. Considera-se o nível de ocorrência de relacionamento, sendo que 1 corresponde a "Fraco" e 7 a "Forte". Assim, quatro questões referente a Cooperação, Confiança, Transferência de Conhecimento e Relações Sociais, buscam identificar se a empresa faz parte ou não de uma rede de cooperação. A descrição das assertivas desse construto, encontra-se no Quadro abaixo.

Quadro 2 - Assertivas do construto Redes de Cooperação

\begin{tabular}{|l|l|}
\hline Dimensão & Item \\
\hline \multirow{4}{*}{ Cooperação } & Existência de cooperação \\
\cline { 2 - 2 } & Existência de confiança \\
\cline { 2 - 2 } & Existência de transferência de conhecimentos \\
\cline { 2 - 2 } & Existência de relações sociais \\
\hline
\end{tabular}

Fonte: Adaptado de Hoffmann et al. (2017).

Após, empregou-se os testes de confiabilidade, como o Alpha de Cronbach (A.C.), Compostite Reability (C.R.) e Average Variance Extracted (A.V.E.). Tanto o Alpha de Cronbach como a Compostite Reability são utilizados para avaliar se a amostra está livre de vieses, ou ainda, se as respostas - em seu conjunto - são confiáveis (RINGLE; SILVA; BIDO, 2014). Já a Average Variance Extracted é a porção dos dados (nas respectivas variáveis) que é explicada por cada um dos constructos, respectivos aos seus conjuntos de variáveis ou quanto, em média, as variáveis se correlacionam positivamente com os seus respectivos constructos (FORNELL; LARCKER, 1981). A Tabela abaixo demonstra os valores obtidos no teste de confiabilidade.

Tabela 1 - Teste de Confiabilidade do construto Redes de Cooperação

\begin{tabular}{c|c|l|l|l}
\hline Construto & Número de Itens & A.C. & C.R. & A.V.E. \\
\hline Redes de Cooperação & 04 & 0,915 & 0,940 & 0,797 \\
\hline
\end{tabular}

Fonte: Dados da Pesquisa (2018).

Verifica-se na Tabela 1 que todos os valores obtidos para o Alpha de Cronbach (A.C.), para a Composite Reliability (C.R.), bem como para a Average Variance Extracted (A.V.E.) foram superiores aos limítrofes indicados pela literatura (Henseler, Ringle e Sinkovics, 2009), que correspondem a >0,60, >0,60 e >0,50 respectivamente. Isso indica que o construto apresenta níveis aceitáveis de confiabilidade. De modo a avaliar a significância das assertivas, foi verificado pelo modo "Bootstrapping" os valores do Teste T de Student, indicados pela literatura valores $\geq 1,96$ (HAIR JR et al., 2014), bem como, o $p$-value $<0,05$. Os resultados estão dispostos na Tabela 2. 
Tabela 2 - Teste T de Student e $p$-value para cada assertiva

\begin{tabular}{c|c|c|c}
\hline Construto & Indicador & Teste T de Student & $\boldsymbol{p}$-value \\
\hline \multirow{3}{*}{ Redes de Cooperação } & Rede_Coop1 & 43,146 & 0,000 \\
\cline { 2 - 4 } & Rede_Coop2 & 33,757 & 0,000 \\
\cline { 2 - 4 } & Rede_Coop3 & 33,517 & 0,000 \\
\cline { 2 - 4 } & Rede_Coop4 & 25,745 & 0,000 \\
\hline
\end{tabular}

Fonte: Dados da Pesquisa (2018).

Dados os valores dispostos na Tabela acima, referente ao Teste T de Student e do $p$ value, observa-se para o Teste T, todos os valores são $\geq 1,96$ (HAIR JR et al., 2014), bem como, os valores de $p$-value < 0,05 para cada assertiva, o que implica em manter todas as assertivas nos seus respectivos construtos.

Por fim, este estudo apresenta abordagem quantitativa, pois esta abordagem é um dos meios de testar as teorias objetivas e examinar a relação que existe entre as variáveis. Essas variáveis podem ser mensuradas por instrumentos para que os dados numéricos possam ser estatisticamente testados (CRESWELL, 2010). Quanto aos objetivos do estudo, enquadra-se como descritiva. Para Hair Jr., et al. (2005) a pesquisa descritiva tem planos estruturados para medir as características da amostra selecionada, que possam ser descritas por meio de uma questão de pesquisa.

A população investigada é composta por empresas de manufatura de todo o Brasil. A amostra foi composta por 125 organizações manufatureiras, de todo segmento e porte, caracterizada como não-probabilística e classificada como amostra por conveniência. $\mathrm{O}$ instrumento de pesquisa utilizado para a coleta de dados foi por meio da aplicação de um questionário on-line composto de 4 questões, com escalas para respostas de 1 a 7 , do tipo Likert.

A coleta dos dados foi realizada por meio da aplicação do questionário ao principal gestor ou responsável pela área de inovação, desenvolvimento de produtos e processos de cada organização, e aplicado entre os meses de junho e setembro de 2018, o que representa corte transversal. Foram realizados contatos diretos por mensagens on line e encaminhamento do questionário eletrônico na plataforma esurv.org disponibilizado gratuitamente na internet. Os dados coletados foram tratados com técnicas de estatística descritiva, conforme segue.

\section{ANÁLISE DOS RESULTADOS}

Esta seção é composta por duas subseções: o perfil da amostra pesquisada e a análise descritiva dos itens mensurados.

\subsection{PERFIL DA AMOSTRA}

A caracterização das organizações respondentes foi realizada por meio da observação da frequência das respostas dispostas na análise descritiva. A primeira parte da análise corresponde ao conhecimento e descrição do perfil dada amostra.

A amostra da pesquisa totalizou em 125 organizações pesquisadas, levantadas por meio de conveniência e conforme a acessibilidade dos pesquisadores. Quanto a classificação das empresas por porte, é possível verificar a distribuição apresentada na Tabela 3. 
Tabela 3 - Distribuição da amostra por porte

\begin{tabular}{c|c|c}
\hline Porte & Percentual & Quantidade \\
\hline Microempresa & $18,4 \%$ & 23 \\
\hline Pequena empresa & $39,2 \%$ & 49 \\
\hline Média empresa & $22,4 \%$ & 28 \\
\hline Grande empresa & $20 \%$ & 25 \\
\hline
\end{tabular}

Fonte: Dados da pesquisa (2018).

Dado a Tabela 3, observa-se que a maior parte das empresas participantes da amostra são classificadas como microempresa e pequena empresa, juntas essas categorias somam 72 organizações, correspondendo um percentual de 57,6\%. Correspondente as médias empresas, 28 organizações compuseram a amostra com um percentual de $22,4 \%$ e, 25 grandes empresas fizeram parte da pesquisa, com percentual de $20 \%$. A distribuição das empresas por porte obedeceu aos critérios de classificação utilizados pelo SEBRAE (2018) e, também recomendados pela OCDE (2005).

A literatura aponta que pequenas e médias empresas tendem a serem mais flexíveis, porém com menor disponibilidade de recursos, quando comparadas às grandes organizações (DAMANPOUR, 1991; GOMES; MACHADO; ALEGRE, 2014). Empresas maiores tendem a serem mais inovadoras que empresas menores, devido a maior disponibilidade de recursos, como em pesquisa e desenvolvimento (MATRAS-BOLIBOK, 2014; GELDES; FELZENSZTEIN; PALACIOS-FENECH, 2017), bem como se mostram mais resistentes à turbulências econômicas (MATRAS-BOLIBOK, 2014).

Com o objetivo de complementar a análise do perfil das organizações respondentes, verificou-se a realização ou não da prática de comércio exterior dentre as organizações respondentes. Os resultados estao apresentados na Tabela 4.

Tabela 4 - Distribuição da amostra por Prática de Comércio Exterior

\begin{tabular}{c|c|c}
\hline Comércio Exterior & Percentual & Quantidade \\
\hline Exporta & $9,6 \%$ & 12 \\
\hline Importa & $11,2 \%$ & 14 \\
\hline Exporta e Importa & $42,4 \%$ & 53 \\
\hline Nenhum & $36,8 \%$ & 46 \\
\hline
\end{tabular}

Fonte: Dados da Pesquisa (2018).

Para esta amostra, verifica-se que dentre as 125 organizações pesquisadas, 46 delas não realizam nenhuma prática de comércio exterior, ou seja, não importam nenhum produto ou matéria-prima, bem como não exportam nenhum de seus produtos, o que corresponde a 36,8\%. Esses valores são expressivos, pois representam mais de um terço da amostra. As atividades de exportação e importação são realizadas por 53 organizações, ou seja, 42,4\% da amostra. Apenas 12 empresas praticam somente atividades de exportação, o que corresponde a menos de $10 \%$ da amostra. 
Em relação ao ramo de atividade das empresas, as mesmas foram classificadas de alta à baixa intensidade tecnológica, conforme suas áreas de atuação. Essa classificação foi baseada em Furtado e Carvalho (2005) e na OCDE (2005), sendo empresas de alta intensidade tecnológica, aquelas que atuam em setores aeroespacial, farmacêutico, de informática, eletrônica e telecomunicações, instrumentos. Organizações caracterizadas como de média-alta intensidade tecnológica, compreendem setores de material elétrico, veículos automotores, química (exceto setor farmacêutico), ferroviário e de equipamentos de transporte, máquinas e equipamentos. Organizações classificadas como média-baixa intensidade tecnológica, se refere aos setores de construção naval, borracha e produtos plásticos, coque, produtos refinados de petróleo e de combustíveis nucleares, outros produtos não metálicos, metalurgia básica e produtos metálicos. Por fim, as empresas de baixa intensidade tecnológica, engloba outros setores e de reciclagem, madeira, papel e celulose, editorial e gráfica, alimentos, bebidas e fumo, têxtil e de confecção, couro e calçados. A distribuição da amostra pesquisada por nível de intensidade tecnológica pode ser verificada na Tabela 5.

Tabela 5 - Distribuição da amostra por nível de intensidade tecnológica

\begin{tabular}{c|c|c}
\hline $\begin{array}{c}\text { Intensidade } \\
\text { Tecnológica }\end{array}$ & Percentual & Quantidade \\
\hline Alta & $0 \%$ & 0 \\
\hline Média alta & $12,8 \%$ & 16 \\
\hline Média baixa & $24,8 \%$ & 31 \\
\hline Baixa & $62,4 \%$ & 78 \\
\hline
\end{tabular}

Fonte: Dados da Pesquisa (2018).

Percebe-se por meio da Tabela 5, que mais de 50\% das organizações que compõem a amostra, caracterizam-se como sendo de baixa intensidade tecnológica, representada por 78 empresas, distribuídas entre os ramos têxtil e de confecção, alimentos, madeira, bebidas e fumo, editorial e gráfica, papel e celulose, reciclagem, couro e calçados. Nesta pesquisa, nenhuma empresa corresponde à classificação de alta intensidade tecnológica. O estudo de Paula e Silva (2017) aponta que empresas de baixa intensidade tecnológica tendem a introduzir mais inovações de processos do que inovações de produtos, enquanto que em organizações de alta tecnologia geralmente ocorre o contrário. Ainda, verifica-se no estudo de Beule e Beveren (2012), que empresas de baixa intensidade tecnológica podem se tornar mais inovadoras quando localizadas em um aglomerado industrial. Zonta et al. (2015) também favorece a crença de que pequenas empresas associadas em rede podem aumentar sua competitividade, colaborando também para o desenvolvimento regional.

Quando questionadas sobre qual o gênero do principal líder da empresa, $80.8 \%$ das empresas possuem os homens à frente das mulheres, que por sua vez, representam somente 19,2\% de liderança nessa amostra. Esses percentuais correspondem a 101 e 24 organizações, respectivamente. É possível verificar no estudo de Cropley e Cropley (2017) que, inicialmente, observa-se uma relação negativa entre a proporção de indivíduos femininos e a capacidade organizacional para inovar (CROPLEY; CROPLEY, 2017). Entretanto, posteriormente verificou-se que a relação entre gênero e capacidade de inovação é complexa e influenciada 
pela cultura organizacional e por fatores individuais, ou seja, o gênero é indiferente nesta relação quando não há ambiente adequado para que a inovação seja bem-sucedida (CROPLEY; CROPLEY, 2017).

\subsection{ANÁLISE DESCRITIVA DOS ITENS MENSURADOS}

A próxima etapa consiste na análise de frequência para cada assertiva mensurada na pesquisa, com o intuito de verificar a distribuição dos dados e o percentual de escolha dos respondentes. Para o rótulo "Fraco" foram somadas as frequências dos pontos 1, 2 e 3 da escala, já correspondente ao rótulo "Forte" foram somados os percentuais obtidos nos pontos 5, 6 e 7 . O ponto 4 da escala foi atribuído como elemento neutro. De forma complementar, também foram calculados média e desvio padrão médio de cada assertiva. Assim, segue dados na Tabela 6.

Tabela 6 - Análise Descritiva das assertivas sobre Redes de Cooperação

\begin{tabular}{|c|c|c|c|c|c|c|c|c|c|c|c|}
\hline \multirow{2}{*}{ Assertiva } & \multicolumn{7}{|c|}{ Frequência em percentual (\%) } & \multicolumn{2}{|c|}{$\begin{array}{l}\text { Ocorrência de } \\
\text { relacionamento }\end{array}$} & \multirow{2}{*}{ Média } & \multirow{2}{*}{$\begin{array}{l}\text { Desvio } \\
\text { Padrão } \\
\text { Médio }\end{array}$} \\
\hline & 1 & 2 & 3 & 4 & 5 & 6 & 7 & Fraco & Forte & & \\
\hline $\begin{array}{l}\text { Qual o nível de } \\
\text { cooperação entre } \\
\text { sua empresa e as } \\
\text { demais empresas da } \\
\text { rede? }\end{array}$ & 3.25 & 5.69 & 10.57 & 7.07 & 28.46 & 26.02 & 8.94 & $19.51 \%$ & $63.42 \%$ & 4,74 & 1,20 \\
\hline $\begin{array}{l}\text { Qual o nível de } \\
\text { confiança entre sua } \\
\text { empresa e as demais } \\
\text { empresas da rede? }\end{array}$ & 2.44 & 1.63 & 9.76 & 16.26 & 24.39 & 30.89 & 14.63 & $13.83 \%$ & $69.91 \%$ & 5,09 & 1,12 \\
\hline $\begin{array}{l}\text { Qual o nível de } \\
\text { transferência de } \\
\text { conhecimento entre } \\
\text { sua empresa e as } \\
\text { demais empresas da } \\
\text { rede? }\end{array}$ & 4.88 & 8.94 & 9.76 & 19.51 & 25.2 & 23.58 & 8.13 & $23.58 \%$ & $56.91 \%$ & 4,54 & 1,33 \\
\hline $\begin{array}{l}\text { Qual o nível das } \\
\text { relações sociais } \\
\text { entre sua empresa e } \\
\text { as demais empresas } \\
\text { da rede? }\end{array}$ & 4.07 & 4.88 & 6.5 & 16.26 & 29.27 & 25.2 & 13.82 & $15.45 \%$ & $68.29 \%$ & 4,91 & 1,16 \\
\hline
\end{tabular}

Fonte: Dados da Pesquisa (2018). 
A análise descritiva, observada por meio da Tabela acima, demonstra que as empresas participantes de redes de cooperação, em sua maioria, apresentam forte ocorrência de relacionamento com outras empresas. Verifica-se que o item confiança é o mais forte dentre os itens, ou seja, as organizações confiam fortemente nas outras. Esse fato é amparado pela literatura, como nos estudos de Felzensztein, Gimmon e Carter (2010), Molina-Morales, Martinez-Fernandez e Torlò (2011), que apontam vantagens como uma maior proximidade entre organizações, que tende a promover um maior compartilhamento de informações, conhecimentos e recursos que são benéficos à organização.

Já o item transferência de conhecimento é o mais fraco dentre os itens relacionados, o que sugere a retenção de conhecimento para a própria organização ou a resistência entre as organizações em compartilharem conhecimentos. Este fato pode ser justificado por meio do estudo de Cantner, Conti e Meder (2010), de que a transferência de conhecimento é algo complexo e caro, visto que o conhecimento tácito não transborda livremente dentro de uma rede. Por essa razão, são necessários esforços específicos para se beneficiar de fontes externas de conhecimento, especialmente em ambientes inovadores (CANTNER; CONTI; MEDER, 2010). Ainda, Gunawan, Jacob e Duysters (2016), apontam que a dependência excessiva do compartilhamento de conhecimento dentro de uma rede pode resultar na difusão de conhecimento redundante, em vez de disponibilizar novos conhecimentos para as organizações envolvidas. No geral, percebe-se que a média de respostas das empresas participantes, ficaram em torno de quatro.

\section{CONSIDERAÇÕES FINAIS}

Este estudo consistiu em desenvolver uma escala quantitativa sobre redes de cooperação, que mensurassem itens como cooperação, confiança, transferência de conhecimentos e relações sociais. A pesquisa, quantitativa, resultou em uma amostra de 125 organizações participantes de redes de cooperação e pertencentes a indústria manufatureira brasileira, levantada por conveniência e acessibilidade.

Após o desenvolvimento da escala, e posterior validação do modelo de mensuração pelos especialistas na área, identificou-se por meio da análise descritiva que a maioria das empresas participantes de redes de cooperação, relacionam-se fortemente mento com outras empresas, no qual item confiança é o mais se destaca, indicando que as organizações confiam fortemente nas outras. Porém a questão relativa à transferência de conhecimento, ocorre com menos intensidade dentre as organizações, o que sugere a retenção de conhecimento para si ou a resistência entre as organizações em compartilharem conhecimentos.

Este estudo contribui em termos de pesquisa por meio do desenvolvimento e a validação de uma escala quantitativa para o construto redes de cooperação, no qual avalia os itens cooperação, confiança, transferência de conhecimento e relações sociais entre as empresas. Sendo que não foi identificado na literatura escala quantitativa para esse construto, esta tornase uma contribuição acadêmica.

Apesar do rigor metodológico adotado, este estudo apresenta algumas limitações que devem ser consideradas na interpretação dos resultados. A começar pela amostra, caracterizada como não probabilística, por conveniência e acessibilidade, impossibilita a generalização dos resultados apresentados neste estudo. Há possibilidade de viés nas respostas pelo fato de somente um representante de cada empresa ter participado da pesquisa, visto que o viés pode 
ocorrer por tratar-se de percepção individual. Para Gomes e Wojahn (2017), o uso de mais de um respondente por empresa poderia aumentar a validade dos dados.

No que se refere às redes de cooperação, o estudo limitou-se a cooperação entre empresas, sem considerar a relação de cooperação que pode dar-se entre empresas e outras instituições, como as universidades e o governo. Assim, como sugestões para pesquisas futuras, sugere-se abranger a utilização da escala para universidades e governo, e comparar como os itens se comportam em cada tipo de instituição. Sugere-se ainda, verificar os itens cooperação, confiança, transferência de conhecimento e relações sociais em ambientes específicos como parques industriais, por exemplo, no qual tem-se também a questão do aglomerado de empresas. Uma última sugestão, seria a realização de um acompanhamento longitudinal, que poderia observar individualmente os itens da escala, e sua variação com o decorrer do tempo, bem como verificar esses itens diante de acontecimentos e turbulências externas.

\section{REFERÊNCIAS}

AHUJA, G. Collaboration networks, structural holes, and innovation: A longitudinal study. Administrative science quarterly, v. 45, n. 3, p. 425-455, 2000.

ANDRADE, H. C. C.; FERREIRA, C. A.; VERONEZE, R. B.; ANTONIALli, F.; CAVAZZA, B. H.; SANTOS, A. C. Estrutura de Governança em Rede e Competitividade no Agronegócio. Revista Espacios, v. 38, n. 26, p. 1-13, 2017.

ANDRIGHI, F. F.; HOFFMANN, V. E.; ANDRADE, M. A. R. Análise da Produção Científica no Campo de Estudo das Redes em Periódicos Nacionais e Internacionais. RAI - Revista de Administração e Inovação, v. 8, n. 2, p. 29-54, 2011.

BALESTRIN, A.; VARGAS, L. M. A Dimensão Estratégica das Redes Horizontais de PMEs: Teorizações e Evidências. RAC - Revista de Administração Contemporânea, v. 8, p. 203 227, 2004.

BEULE, F.; BEVEREN, I. V. Does firm agglomeration drive product innovation and renewal? An application for Belgium. Tijdschrift voor Economische en Sociale Geografi, v. 103, n. 4, p. $457-472,2012$.

CANTNER, U.; CONTI, E.; MEDER, A. Networks and Innovation: The Role of Social Assets in Explaining Firms' Innovative Capacity. European Planning Studies, v. 18, n. 12, p. 19371956, 2010.

CONSENTINO, C. G.; CARVAlHO, R. P. DE; GOULART, I. B. Formação e desenvolvimento de redes formais de micro e pequenas empresas: Um estudo de caso de uma sociedade de propósito específico. Revista Espacios, v. 38, n. 28, p. 1-14, 2017.

CRESWELL, John W. Projeto de pesquisa: métodos qualitativo, quantitativo e misto.5. ed. Porto Alegre: Artmed: Bookman, 2010. 
CROPLEY, D.; CROPLEY, A. Innovation Capacity , Organisational Culture and Gender. European Journal of Innovation Management, p. 1-34, 2017.

CUNHA, J. A. C. DA; PASSADOR, J. L.; PASSADOR, C. S. Recomendações e apontamentos para categorizações em pesquisas sobre redes interorganizacionais. Cadernos EBAPE.BR, v. 9, n. Edição especial, artigo 4, 2012.

DAMANPOUR, F. Organizational Innovation: A meta-analysis of effects of determinants and moderators. Academy of Management Journal, v. 34, n. 3, p. 555-590, 1991.

DAMANPOUR, F.; SZABAT, K.; EVAN, W. The relationship between types of innovation and organizational performance. Journal of Management Studies, v. 26, n. 6, p. 587-601, 1989.

DIAS, C.; FRANCO, M. Cooperation in tradition or tradition in cooperation? Networks of agricultural entrepreneurs. Land Use Policy, v. 71, p. 36-48, 2018.

FELZENSZTEIN, C.; GIMMON, E.; CARTER, S. Geographical co-location, social networks and inter-firm marketing co-operation: the case of the salmon industry. Long Range Planning, v. 43, n. 5/6, p. 675-690, 2010.

FORNELL, C.; LARCKER, D. F. Evaluating structural equation models with unobservable variables and measurement error. Journal of Marketing Research, p. 39-50, 1981.

FRANCO, M.; BELO, M. B. Cooperation networks as a mechanism for strengthening territorial competitiveness: the case of the Qualifica Association. World Review of Entrepreneurship, Management and Sustainable Development, v. 9, n. 4, p. 421-443, 2013.

FURTADO, A. T.; CARVALHO, R. Q. Padrões de intensidade tecnológica da indústria brasileira um estudo comparativo com os países centrais. São Paulo em Perspectiva, v. 19, n. 1, p. 70-84, 2005.

GELDES, C.; FELZENSZTEIN, C.; PALACIOS-FENECH, J. Technological and nontechnological innovations, performance and propensity to innovate across industries: The case of an emerging economy. Industrial Marketing Management, v. 61, p. 55-66, 2017.

GOMES, G.; MACHADO, D. D. P. N.; ALEGRE, J. Indústria têxtil de Santa Catarina e sua capacidade inovadora: Estudo sob a perspectiva da eficiência, eficácia, custos e melhoria de processos. RAI - Revista de Administração e Inovação, v. 11, n. 2, p. 273-294, 2014.

GOMES, G.; WOJAHN, R. M. Organizational learning capability, innovation and performance: study in small and medium-sized enterprises (SMES). RAUSP Management Journal, v. 52, n. 2, p. 163-175, 2017. 
GRANDORI, A.; SODA, G. Inter-firm Networks: Antecedents, Mechanisms and Forms. Organization Studies, v. 16, n. 2, p. 183-214, 1995.

GULATI, R. Alliances and networks. Strategic management journal, v. 19, n. 4, p. 293-317, 1998.

GUNAWAN, T.; JACOB, J.; DUYSTERS, G. Network ties and entrepreneurial orientation: Innovative performance of SMEs in a developing country. International Entrepreneurship and Management Journal, v. 12, n. 2, p. 575-599, 2016.

HAIR JR, J. F.; ANDERSON, R. E.; TATHAM, R. L.; BLACK, W. C. Análise multivariada de dados. $5^{\text {a }}$ ed. Porto Alegre: Bookmann, 2005a.

HAIR JR, J. F.; HULT, G. T. M.; RINGLE, C. M. SARSTEDT, M. A primer on partial least squares structural equation modeling (PLS-SEM). Sage Publications, 2014.

HENSELER, J.; RINGLE, C. M.; SINKOVICS, R. R. The use of partial least squares path modeling in international marketing. Advances in International Marketing (AIM), v. 20, p. 277-320, 2009.

HOFFMANN, V. E.; BELUSSI, F. MARTÍNEZ-FERNÁNDEZ, M. T.; REYES JR., E. United we stand, divided we fall? Clustered firms' relationships after the 2008 crisis. Entrepreneurship \& Regional Development, p. 1-24, 2017.

HOFFMANN, V. E.; LOPES, G. S. C.; MEDEIROS, J. J. Knowledge transfer among the small businesses of a Brazilian cluster. Journal of Business Research, v. 67, p. 856-864, 2014.

HOFMANN, V. E.; BANDEIRA-DE-MELLO, R.; MOLINA-MORALES, F. X. Innovation and Knowledge Transfer in Clustered Interorganizational Networks in Brazil. Routledge Taylor \& Francis Group, v. 12, p. 143-163, 2011.

HOSKISSON, R. E. et al. Theory and research in strategic management: swings of a pendulum. Journal of Management, v. 25, n. 3, 1999.

JÚNIOR, N. DA S. M.; COSTA, H. A.; HOFFMANN, V. E. Dificuldades para a Cooperação entre Hotéis Aglomerados Territorialmente: um estudo da hotelaria em Brasília - DF. Turismo em Análise, v. 27, n. 1, p. 153-177, 2016.

MATRAS-BOLIBOK, A. Does firm's size impact innovative performance? International Journal of Innovation and Learning, v. 15, n. 4, p. 422-431, 2014.

MOLINA-MORALES, F. X.; BELSO-MARTÍNEZ, J.; MÁS-VERDÚ, F.; MARTÍNEZCHÁFER, L. Formation and dissolution of inter- fi rm linkages in lengthy and stable networks 
in clusters. Journal of Business Research, v. 68, n. 7, p. 1557-1562, 2015.

MOLINA-MORALES, F. X.; MARTÍNEZ-FERNÁNDEZ, M. T. Redes de Empresas: Proposta de uma Tipologia para Classificaçăo Aplicada na Indústria de Cerâmica. RAC Revista de Administração Contemporânea, v. $1^{a}$ Edição, p. 103-127, 2007.

MOLINA-MORALES, F. X.; MARTINEZ-FERNANDEZ, M. T. Research notes and commentaries too much love in the neighborhood can hurt: How an excess of intensity and trust in relationships may produce negative. Strategic Management Journal, v. 30, p. 1013-1023, 2009.

MOLINA-MORALES, F. X.; MARTINEZ-FERNANDEZ, M. T.; TORLÒ, V. J. The Dark Side of Trust : The Benefits, Costs and Optimal Levels of Trust for Innovation Performance. Long Range Planning, v. 44, p. 118-133, 2011.

OECD, Organização para Cooperação e Desenvolvimento Econômico. Manual de Oslo: Diretrizes para coleta e interpretação de dados sobre inovação, FINEP. 3 ed. 2005.

OLAVE, M. E. L.; NETO, J. A. Redes de Cooperação Produtiva: Uma estratégia de competitividade e sobrevivência para pequenas e médias empresas. Gestão \& Produção, v. 8, n. 3, p. 289-303, 2001.

PAULA, F. D. O.; SILVA, J. F. DA. Innovation performance of Italian manufacturing firms: The effect of internal and external knowledge sources. European Journal of Innovation Management, v. 20, n. 3, p. 428-445, 2017.

POWELL, W. Neither Market for Hierarchy network forms of organization. Research in Organizational Behavior, v. 12, p. 295-336, 1990.

POWELL, W. W.; KOPUT, K. W.; SMITH-DOERR, L. Interorganizational collaboration and the locus of innovation: Networks of learning in biotechnology. Administrative science quarterly, p. 116-145, 1996.

PROVAN, K. G.; FISH, A.; SYDOW, J. Interorganizational networks at the network level: A review of the empirical literature on whole natworks. Journal of Management, v. 33, n. 3, p. 479-516, 2007.

RINGLE, C. M.; SILVA, D. DA; BIDO, D. Modelagem de Equações Estruturais com utilização do SmartPLS. REMark - Revista Brasileira de Marketing, v. 13, n. 2, p. 56-73, 2014.

ROLT, C. R. DE; DIAS, J. DA S.; PEÑA, F. T. G. Network analysis as a management tool for inter-organizational projects. Gestão \& Produção, v. 24, n. 2, p. 266-278, 2017. 
SEBRAE. Critérios de classificação de empresas: EI - ME - EPP. [2018]. Disponível em < http://www.sebrae-sc.com.br/leis/default.asp?vcdtexto=4154>. Acesso em 20 mar. 2018.

STROBEL, N.; KRATZER, J. Obstacles to innovation for SMEs: Evidence from Germany. International Journal of Innovation Management, v. 21, n. 3, p. 1-28, 2017.

THORGREN, S.; WINCENT, J.; ÖRTQVIST, D. Designing interorganizational networks for innovation: An empirical examination of network configuration, formation and governance. Journal of Engineering and Technology Management, v. 26, n. 3, p. 148-166, 2009.

VERSCHOORE, J.; BALESTRIN, A.; TEIXEIRA, R. Network management and associated firms' outcomes: multilevel analysis in the southern Brazilian context. Journal of Management \& Governance, v. 21, n. 1, p. 211-232, 2017.

WERNERFELT, B. A Resource-Based View of the Firm. Strategic Management Journal, v. 5, n. 2, p. 171-180, 1984.

YOU, W.; SHU, H.; LUO, S. Competition, cooperation, and performance: an empirical investigation of Chinese online sellers. Information Systems and e-Business Management, v. 16, n. 4, 2017.

ZONTA, P. C., MOLOZZI, G.; JENTZ, J; CARVALHO, C. E. Relação entre Cooperação e Aprendizado Organizacional com a Competitividade em uma Rede Interorganizacional. REDES, v. 20, n. 1, p. 179-193, 2015. 